\title{
Electromagnetic Wave Equations of Dyon in Arbitrary Media
}

\author{
I. Joshi" , J. S. Garia \\ Department of Physics, L. S. M. Govt. P.G. College, Pithoragarh-U.K. \\ Received 5 September 2019, accepted in final revised form 26 December 2019
}

\begin{abstract}
Dyon is a hypothetical particle in high energy physics that carries simultaneously both electric and magnetic charge. A dyon with zero electric charge is referred to a magnetic monopole. The paper, reports a simple reformulation of Maxwell equations for dyon in arbitrary media. The Lorentz, Coulomb gauge conditions and the wave equations of dyon in arbitrary media are derived in a simple and compact manner.
\end{abstract}

Keywords: Dyon; Electromagnetic fields; Wave Equation; Polarization; Magnetization.

() 2020 JSR Publications. ISSN: 2070-0237 (Print); 2070-0245 (Online). All rights reserved.

doi: http://dx.doi.org/10.3329/jsr.v12i2.43635

J. Sci. Res. 12 (2), 161-167 (2020)

\section{Introduction}

The question of existence of monopole [1] and dyons [2-11] has become a challenging new frontier and the object of more interest in the recent years of high energy physics. Dirac revealed that a single magnetic pole anywhere in the universe would explain the quantization of electric charge. Schwinger-Zwanziger [12,13] provided the generalization of this quantization condition for dyons. Keeping in view the result of witten that monopole are necessarily dyons. Magnetic monopoles have not been seen in the real world. Defining certain physical quantities including magnetic monopole is difficult. Therefore, the symmetry between electric and magnetic charge is very useful in describing these quantities. The experimental appearance of composite entities whose behavior is similar to that of the magnetic monopoles is the main cause for establishing new electrodynamics within the solid, in which there are magnetic fields whose divergence is different from zero. The possible generalized approach that derives to these equations was analyzed with the help of dyon as a particle with electric and magnetic charge. According to this approach, the charge description can be set in a complex plane. The electric charge is placed on the real axis, and the magnetic charge is represented on the imaginary axis. Keeping in view the recent potential importance of monopoles and dyons along with the fact that despite the potential importance of monopoles, the formalism necessary to describe them has been clumsy and not manifestly covariant, Negi

Corresponding author: $\underline{\text { ilajoshi0090@gmail.com }}$ 
and coworkers has already developed a self-consistent quantum field theory of generalized electromagnetic fields associated with dyons (particles carrying electric and magnetic charges) [14-16]. Most of the researches previously reported Maxwell equation of dyon [14,15], in different media (homogenous, chiral medium, isotropic) medium). The four Maxwell equations, whose formulation in the emptyspace [17] (the vacuum) are already well known, have been reconstructed for the existence of matter with electric and magnetic charges. Jefimenko [18] has derived the solution of the Maxwell equation in arbitrary media (dielectric and magnetic media) in the absence of monopole. This paper considers Maxwell equations of dyon within matter. The Maxwell equations for dyon in arbitrary media (dielectric and magnetic media) are derived [19]. In this paper, we have made an attempt to show the wave equations for dyon in the case of separate electric and magnetic charge in arbitrary media.

\section{Field Associated with Dyon}

Considering the existence of of magnetic monople Dirac [20] generalised Maxwell field equation for dyon in following manner in vaccum [21-23] in SI units for $\mathrm{c}=\hbar=1$ as,

$$
\begin{aligned}
\vec{\nabla} \cdot \vec{E} & =\frac{\rho_{e}}{\varepsilon} \\
\vec{\nabla} \cdot \vec{B} & =\mu \rho_{m} \\
\vec{\nabla} \times \vec{E} & =-\frac{\partial \vec{B}}{\partial t}-\frac{j_{m}}{\varepsilon} \\
\vec{\nabla} \times \vec{B} & =\mu j_{e}+\frac{\partial \vec{E}}{\partial t}
\end{aligned}
$$

The electric and magnetic field associated with dyons carrying generalized charge can be written as [22]

$q=e-i g$

Where $e$ and $g$, are electric and magnetic charges respectively. The generalized four potentials associated with dyons are defined as,

$V_{\mu}=C_{\mu}-i D_{\mu}$

Where $C_{\mu}$ and $D_{\mu}$ are electric and magnetic four potentials respectively. Electric and magnetic fields of dyons in terms of components of electric and magnetic potentials are defined as,

$$
\begin{aligned}
& \vec{E}=-\vec{\nabla} \phi_{e}-\frac{\partial \vec{C}}{\partial t}-\vec{\nabla} \times \vec{D} \\
& \vec{B}=-\vec{\nabla} \phi_{m}-\frac{\partial \vec{D}}{\partial t}+\vec{\nabla} \times \vec{C}
\end{aligned}
$$


The vector wave function $\psi$ associated with generalized electromagnetic fields is defined as,

$$
\vec{\psi}=\vec{E}-i \vec{B}
$$

We get following the form of generalized Maxwell's equations for dyons

$$
\begin{aligned}
& \vec{\nabla} \cdot \vec{\psi}=\frac{\rho}{\varepsilon_{0}} \\
& \vec{\nabla} \cdot \vec{\psi}=\frac{\rho}{\varepsilon_{0}}
\end{aligned}
$$

Where $J=(\rho, j), \rho$ and $\mathrm{j}$ are the generalized charge and current source densities of dyons given by

$\rho=\rho_{e}-i \rho_{m}$

$\vec{j}=\vec{j}_{e}-\overrightarrow{i j}_{m}$

The generalized covariant form of Maxwell Dirac equations of dyon as,

$$
\begin{aligned}
& \partial_{\mu}\left(F^{\mu v}\right)=\mu j^{v(e)} \\
& \partial_{\mu}\left(F_{d}{ }^{\mu v}\right)=\mu j^{v(m)}
\end{aligned}
$$

The generalized field equation of dyon is given as

$G_{\mu v, v}=j_{\mu}$

Where $J_{\mu}=(\rho,-J)$

\section{Maxwell Field Equation of Dyon in Arbitrary Media}

Considering the existence of magnetic charge and current density, in addition to the electric charge and current density, the Maxwell equations of dyon in arbitrary media would be [19]

$$
\begin{aligned}
& \vec{\nabla} \cdot \vec{E}=\frac{1}{\varepsilon_{0}}\left(\rho_{e}+\rho_{P}\right) \\
& \vec{\nabla} \cdot \vec{H}=\rho_{m}+\frac{\rho_{M}}{\mu_{0}} \\
& \vec{\nabla} \times \vec{D}=\varepsilon_{0} J_{P}-\varepsilon_{0} \frac{\partial M}{\partial t}-\frac{\partial H}{\partial t}-j_{m} \\
& \nabla \times \vec{B}=\mu_{0} J_{M}+\mu_{0} j_{e}+\frac{\partial E}{\partial t}+\mu_{0} \frac{\partial P}{\partial t}
\end{aligned}
$$

By using $\left(c^{2}=\frac{1}{\mu_{0} \varepsilon_{0}}, c=\hbar=1\right)$, Maxwell's field equation of dyon in terms of $j_{e}, j_{m}, \mathrm{~J}_{\mathrm{M}}$, $M$ and $P$ in arbitrary media are derived. The electric field, magnetic field, displacement field and magnetic intensity vector of dyon are defined in terms of components of potentials as [19].

$$
\vec{E}=-\nabla \Phi_{e}-\frac{\partial \vec{C}}{\partial t}-\vec{\nabla} \times \vec{D}
$$


$\vec{B}=-\vec{\nabla} \phi_{m}-\frac{\partial D}{\partial t}+\nabla \times C$

$\vec{D}=-\nabla \Phi_{D}-\frac{\partial \vec{G}}{\partial t}-\vec{\nabla} \times \vec{I}$

$\vec{H}=-\vec{\nabla} \Phi_{H}-\frac{\partial \vec{I}}{\partial t}+\vec{\nabla} \times \vec{G}$

Where $\phi_{e}, \phi_{m}, \phi_{D}$ and $\phi_{H}$ are scalar potential and $C, D, G$ and $I$ are vector potential for electric and magnetic charge in case of electric field, magnetic field, displacement field and magnetic intensity vector respectively.

\section{Potential Formulation of Dyon}

Putting the value of $E, B, D$ and $H$ into the equations (15-18), after solving these equations we get scalar and vector wave equations for electric and magnetic charge in arbitrary media respectively as,

$$
\begin{aligned}
& \nabla^{2} \Phi_{e}+\frac{\partial}{\partial t}(\nabla \cdot C)=-\frac{\rho_{e}+\rho_{P}}{\varepsilon_{0}} \\
& \nabla^{2} \Phi_{H}+\frac{\partial(\nabla \cdot \vec{I})}{\partial t}=-\left(\rho_{m}+\varepsilon_{0} \rho_{M}\right) \\
& \frac{\partial^{2} I}{\partial t}-\nabla^{2} I+\nabla\left(\nabla \cdot I+\frac{\partial \Phi_{H}}{\partial t}\right)= \\
& -\varepsilon_{0} J_{p}+\varepsilon_{0} \frac{\partial M}{\partial t}+j_{m} \quad \\
& \frac{\partial^{2} C}{\partial t^{2}}-\nabla^{2} C+\nabla\left(\nabla \cdot C+\frac{\partial \phi_{e}}{\partial t}\right)=\mu_{0} J_{M}+\mu_{0} j_{e} \\
& \quad+\mu_{0} \frac{\partial P}{\partial t}
\end{aligned}
$$

\section{Coulomb Gauge}

The coulomb gauge condition for vector potential in case of electric field and magnetic intensity respectively as,

$\vec{\nabla} \cdot \vec{C}=0$

$\vec{\nabla} \cdot \vec{I}=0$

Then the inhomogenous wave equations (23-26) can be written as,

$$
\begin{aligned}
& \nabla^{2} \phi_{e}=-\frac{\rho_{e}+\rho_{P}}{\varepsilon_{0}} \\
& \nabla^{2} \phi_{H}=-\left(\rho_{m}+\frac{\rho_{M}}{\mu_{0}}\right) \\
& \nabla^{2} I-\frac{\partial^{2} I}{\partial t^{2}}=-\varepsilon_{0} J_{p}+j_{m}+\varepsilon_{0} \frac{\partial M}{\partial t}-\nabla \frac{\partial \Phi_{H}}{\partial t}
\end{aligned}
$$


$\nabla^{2} C-\frac{\partial^{2} C}{\partial t^{2}}=\mu_{0} J_{M}+\mu_{0} j_{e}+\mu_{0} \frac{\partial P}{\partial t}-\nabla \frac{\partial}{\partial t} \phi_{e}$

Equations (29-32) are the time-dependent Poisson's equations which lead to the following solutions as,

$$
\begin{aligned}
& \Phi_{e}=\frac{1}{4 \pi \varepsilon_{0}} \int_{v} d^{3} x \frac{\rho_{e}+\rho_{P}\left(t^{\prime}{ }_{r e t}, x^{\prime}\right)}{x-x^{\prime}}+\alpha \\
& \Phi_{H}=\frac{1}{4 \pi \mu_{0}} \int_{v}^{1} d^{3} x \frac{\mu_{0} \rho_{m}+\rho_{M}\left(t^{\prime}{ }_{r e t}, x^{\prime}\right)}{x-x^{\prime}}+\alpha
\end{aligned}
$$

Where $\alpha$ has vanishing gradient.

By putting the value of electric and magnetic scalar potentials for electric field and magnetic intensity vector, inhomogenous wave equations (33) and (34), are obtained as,

$$
\begin{aligned}
\nabla^{2} I-\frac{\partial^{2} I}{\partial t^{2}} & =-\varepsilon_{0} J_{P}+\vec{j}_{m}+\varepsilon_{0} \frac{\partial M}{\partial t} \\
- & \frac{1}{4 \pi \mu_{0}} \nabla \frac{\partial}{\partial t} \int_{v} d^{3} x \frac{\left(\mu_{0} \rho_{m}+\rho_{M}\right)\left(t_{r e t}^{\prime}, x^{\prime}\right)}{x-x^{\prime}} \\
\nabla^{2} C-\frac{\partial^{2} C}{\partial t^{2}} & =\mu_{0} J_{M}+\mu_{0} \vec{j}_{e}+\mu_{0} \frac{\partial P}{\partial t} \\
& -\frac{1}{4 \pi \varepsilon_{0}} \nabla \int_{v} d^{3} x \frac{\left(\rho_{e}+\rho_{P}\right)\left(t^{\prime}{ }_{r e t}, x^{\prime}\right)}{x-x^{\prime}}
\end{aligned}
$$

\section{Lorentz Gauge}

The Lorentz gauge condition for vector potential in case of electric field and magnetic intensity respectively as,

$$
\begin{aligned}
& \vec{\nabla} \cdot \vec{I}+\frac{\partial \Phi_{H}}{\partial t}=0 \\
& \vec{\nabla} \cdot \vec{C}+\frac{\partial \phi_{e}}{\partial t}=0
\end{aligned}
$$

Then the inhomogenous wave equations (23-26) can be written as,

$$
\begin{aligned}
& \frac{\partial^{2} \phi_{e}}{\partial t^{2}}-\nabla^{2} \phi_{e}=\frac{\rho_{e}+\rho_{P}}{\varepsilon_{0}} \\
& \frac{\partial^{2} \phi_{H}}{\partial t^{2}}-\nabla^{2} \phi_{H}=\left(\rho_{m}+\frac{\rho_{M}}{\mu_{0}}\right) \\
& \frac{\partial^{2} I}{\partial t^{2}}-\nabla^{2} I=-\varepsilon_{0} J_{P}+\vec{j}_{m}+\varepsilon_{0} \frac{\partial M}{\partial t} \\
& \frac{\partial^{2} C}{\partial t^{2}}-\nabla^{2} C=\mu_{0} J_{M}+\mu_{0} j_{e}+\mu_{0} \frac{\partial P}{\partial t}
\end{aligned}
$$

Under the assumption of causality, the solutions of equation (39-42) are retarted potentials as, 


$$
\begin{aligned}
& \phi_{e}=\frac{1}{4 \pi \varepsilon_{0}} \int_{v} d^{3} x^{\prime} \frac{\left(\rho_{e}+\rho_{P}\right)\left(t_{r e t}^{\prime}, x^{\prime}\right)}{x-x^{\prime}} \\
& \phi_{H}=\frac{1}{4 \pi \mu_{0}} \int_{v} d^{3} x^{\prime} \frac{\left(\mu_{0} \rho_{m}+\rho_{M}\right)\left(t_{r e t}^{\prime}, x^{\prime}\right)}{x-x^{\prime}} \\
& I=\frac{1}{4 \pi} \int_{v} d^{3} x^{\prime} \frac{\left(-\varepsilon_{0} J_{p}+j_{m}+\varepsilon_{0} \frac{\partial M}{\partial t}\right)\left(t_{r e t}^{\prime}, x^{\prime}\right)}{x-x^{\prime}} \\
& C=\frac{1}{4 \pi} \int_{v} d^{3} x^{\prime} \frac{\left(\mu_{0} \frac{\partial P}{\partial t}\right)\left(t_{r e t}^{\prime}, x^{\prime}\right)}{x-x^{\prime}}
\end{aligned}
$$

\section{Conclusion}

In this paper, we have discussed the field associated with dyon, Maxwell field equations of dyon and the wave equations of dyon in arbitrary media. The wave equations for electric and magnetic charge in case of electric field and magnetic intensity are derived in arbitrary media. The generalized electromagnetic wave equation of dyon associated with Lorentz and Coulomb gauge are also derived and their solutions of inhomogenous wave equations are deduced. These equations reduce to the theory of electric (magnetic charge) in the absence of magnetic charge on dyon or vice-versa.

\section{References}

1. P. A. M. Dirac, Proc. Roy. Soc. A133, 60 (1931).

https://royalsocietypublishing.org/doi/abs/10.1098/rspa.1931.0130

2. G.' t Hooft, Nucl. Phys. B79, 276 (1974).

https://www.sciencedirect.com/science/article/pii/0550321374904866

3. A. M. Polyakov, JETP Lett. 20, 194 (1974).

http://www.jetpletters.ac.ru/ps/1789/article 27297.shtml

4. J. Schwinger, Phys. Rev. 144, 1087 (1966).

https://journals.aps.org/pr/abstract/10.1103/PhysRev.144.1087

5. J. Schwinger, Phys. Rev. 151, 1048 (1966).

6. J. Schwinger, Phys. Rev. 151, 1055 (1966). https://journals.aps.org/pr/abstract/10.1103/PhysRev.151.1055

7. J. Schwinger, Science 165, 757 (1969). https://science.sciencemag.org/content/165/3895/757

8. D. Zwanziger, Phys. Rev. B 137, 647 (1965). https://journals.aps.org/pr/abstract/10.1103/PhysRev.137.B647

9. D. Zwanziger, Phys. Rev.176, 1489 (1968). https://journals.aps.org/pr/abstract/10.1103/PhysRev.176.1489

10. B. Julia and A. Zee, Phys. Rev. D21, 2940 (1980). http://inspirehep.net/record/143579? ln=en

11. M. Prasad and C. Sommerfield, Phys. Rev. Lett. 35, 760 (1975). https://journals.aps.org/prl/abstract/10.1103/PhysRevLett.35.760

12. J. Schwinger, Phys. Rev. 144, 1087 (1966). http://cgl.soic.indiana.edu/jpac/QCDRef/1960s/Magnetic\%20Charge\%20and\%20Quantum\%20 Field\%20Theory\%20-\%20Schwinger\%20-\%201966.pdf 
13. D. Zwanziger, Phys. Rev. 176, 1489 (1968). http://inspirehep.net/record/52804? ln=en

14. P. S. Bisht, O. P. S. Negi, and B. S. Rajput, Ind. J. Pure Appl. Phys. 24, 543 (1993). https://insa.nic.in/writereaddata/UpLoadedFiles/IJPAM/20005a57_543.pdf

15. J. Singh, P. S. Bisht, and O. P. S. Negi, J. Phys. A: Math. Theor. 40, 11395 (2007). https://iopscience.iop.org/article/10.1088/1751-8113/40/37/015/meta

16. J. Singh, P. S. Bisht, and O. P. S. Negi, J. Phys. A: Math. Theor. 40, 9137 (2007). https://iopscience.iop.org/article/10.1088/1751-8113/40/30/031/meta

17. D. J. Griffiths and M. A. Heald, Am. J. Phys. 59, 111 (1991).

18. O. D. Jefimenko, Am. J. Phys. 60, 899 (1992).

19. I. Joshi and J. S. Garia, Ind. J. Phys. (2019). https://link.springer.com/article/10.1007\%2Fs12648-019-01623-3

20. P. A. M. Dirac, Phys. Rev. 74, 817 (1948). https://journals.aps.org/pr/abstract/10.1103/PhysRev.74.817

21. B. Julia and A. Zee, Phys. Rev. D11, 2227 (1975). https://journals.aps.org/prd/abstract/10.1103/PhysRevD.11.2227

22. P. S. Bisht, O. P. S. Negi, and B. S, Rajput, Int. J. Theor. Phys. 32, 2099 (1993). https://link.springer.com/article/10.1007/BF00675023

23. B. S. Rajput and D. C. Joshi, Hadronic J. 4, 1805 (1981). 